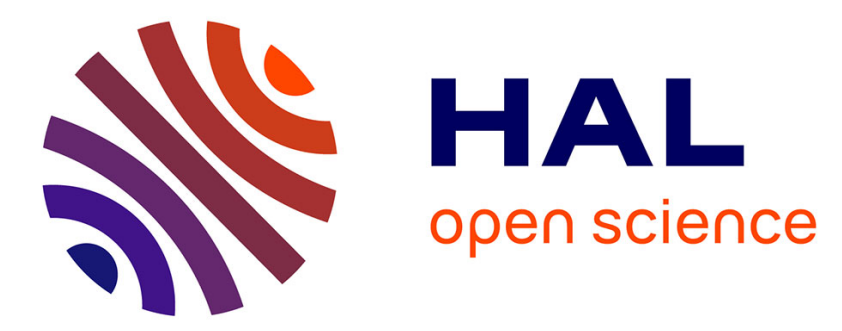

\title{
Les débats sur les fondements de la perspective linéaire de Piero della Francesca à Egnatio Danti : un cas de mathématisation à rebours
}

Dominique Raynaud

\section{To cite this version:}

Dominique Raynaud. Les débats sur les fondements de la perspective linéaire de Piero della Francesca à Egnatio Danti : un cas de mathématisation à rebours. 2008. halshs-00479820

\section{HAL Id: halshs-00479820 \\ https://shs.hal.science/halshs-00479820}

Preprint submitted on 3 May 2010

HAL is a multi-disciplinary open access archive for the deposit and dissemination of scientific research documents, whether they are published or not. The documents may come from teaching and research institutions in France or abroad, or from public or private research centers.
L'archive ouverte pluridisciplinaire HAL, est destinée au dépôt et à la diffusion de documents scientifiques de niveau recherche, publiés ou non, émanant des établissements d'enseignement et de recherche français ou étrangers, des laboratoires publics ou privés. 


\title{
Les débats sur les fondements de la perspective linéaire de Piero della Francesca à Egnatio Danti : un cas de mathématisation à rebours
}

\author{
Dominique Raynaud, PLC (Grenoble), GEMASS (Paris)
}

\begin{abstract}
RESUME. L'essor de la perspective linéaire a suscité de nombreuses polémiques tout au long du Quattrocento et du Cinquecento, opposant les partisans d'une géométrisation artificialiste de la vision à ceux qui vantaient les qualités du dessin d'après nature ou invoquaient des arguments de nature physiologique. Ces débats peuvent être retracés à partir des quatre alternatives qui en constituent le noyau dur : champ de vision restreint vs. large ; immobilité vs. mobilité oculaire; tableau plan vs. curviligne ; vision monoculaire vs. binoculaire. En retenant les premiers termes de ces quatre alternatives, l'histoire de la perspective a rejeté de nombreux systèmes hétérodoxes. Du point de vue de la mathématisation, l'intérêt de ces débats tient à ce qu'ils ont succédé, et non précédé, l'adoption du dispositif perspectif comme intersection de la pyramide visuelle. L'histoire de la perspective linéaire offre ainsi un authentique cas de justification a posteriori des fondements ou, pour ainsi dire, de mathématisation à rebours.
\end{abstract}

MOTS-CLEFS. Mathématisation, géométrie, perspective linéaire, $\mathrm{XV}^{\mathrm{e}}-\mathrm{XVI}^{\mathrm{e}}$ siècles.

ABSTRACT. The debates on the foundations of linear perspective from Piero della Francesca to Egnatio Danti. A case of upside down mathematization. The rise of linear perspective caused many polemics throughout quattrocento and cinquecento, opposing the supporters of artificial geometrisation of sight to those who were praising the qualities of the drawing according to nature, or were invoking some arguments on physiological basis. These debates can be recalled starting from the four alternatives that form the hard core of them: restricted vs. broad field of vision, ocular immobility vs. mobility; curvilinear vs. planar picture, monocular vs. binocular vision. By retaining the first terms of these four alternatives, the history of perspective eliminated many heterodox constructions. From the mathematisation viewpoint, the interest of these debates is that they have succeeded, not preceded, the adoption of perspective system, defined by the intersection of the visual pyramid. Thus the history of linear perspective constitutes a genuine case of a posteriori justification of foundations or so to speak: an upside down mathematisation.

KEYWORDS. Mathematisation, Geometry, Linear perspective, $\mathrm{XV}^{\mathrm{th}}-\mathrm{XVI}^{\mathrm{th}}$ centuries.

\section{MATHÉMATISATION/GÉOMÉTRISATION}

Mathématiser c'est axiomatiser, formaliser, quantifier ou introduire des concepts mathématiques, propres ou importés. La quatrième composante de cette définition est triviale. Les trois premières permettent de rendre compte du mouvement de transformation de nombreuses doctrines informes, mais elles s'ajustent mal à certaines sciences, notoirement aux sciences géométriques.

L'axiomatisation, la formalisation et la quantification sont des aspects possibles, mais toujours contingents, du raisonnement géométrique. Pour preuve certains théorèmes d'unicité ou d'impossibilité qui, bien qu'indirectement rapportés à la trame des Éléments, répondent imparfaitement à la définition de la mathématisation. Prenons la Rédaction des Éléments d'Euclide attribuée à Nasîr al-Dîn al-Tûsî, prop. I, 1: "Sur une droite limitée donnée, construire un triangle équilatéral". Euclide ayant construit deux cercles de centres $A$ et $B$ de même rayon $A B=B A$ (Figure 1), Tûsî introduit la glose suivante: "La circonférence de l'un des deux cercles doit couper la circonférence de l'autre, sinon son centre se situerait sur, ou à l'extérieur, de sa circonférence; ce qui est absurde." 


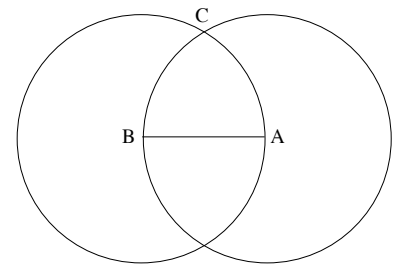

Figure 1

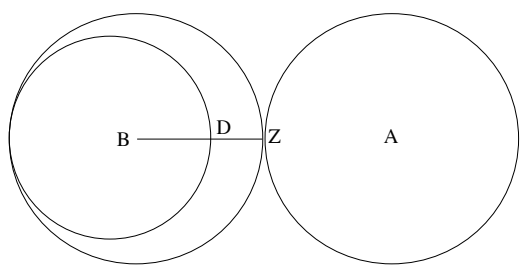

Figure 2

Supposons que les deux cercles ne se coupent pas (Figure 2). Si le cercle $B$ est tangent au cercle $A$, alors son centre est sur sa circonférence, $A=Z$. Si le cercle $B$ n'est pas tangent au cercle $A$, alors son centre est à l'extérieur de sa circonférence, $A=D$, ce qui est absurde, puisque le centre d'un cercle est à l'intérieur de la circonférence. Les cercles sont donc sécants. La démonstration se fonde sur des caractères topologiques (intérieur, extérieur, frontière), absents de l'axiomatique des Éléments. ${ }^{2}$ Il s'ensuit que la démonstration est conduite en dehors de toute axiomatisation. Étant une démonstration géométrique, elle ne recourt pas à la formalisation. Enfin, les concepts topologiques ne présupposant pas une mesure, la démonstration n'appelle pas de quantification. Pourtant, il est indubitable que la démonstration de Tûsî relève d'une activité de mathématisation. Pour la qualifier, il ne reste que la définition triviale de la mathématisation comme usage d'objets ou de concepts mathématiques. Mais celle-là est imprécise, car des concepts mathématiques ont parfois été utilisés en dehors de toute mathématisation. ${ }^{3}$ L'intérêt principal de la glose de Tûsî est donc qu'elle incite à spécifier la mathématisation comme activité démonstrative. ${ }^{4}$

L'argument développé dans cet article est que, parmi les diverses formes que peut prendre la mathématisation, il en est une, trop peu étudiée, qui consiste à «mathématiserà rebours » un domaine. Dans l'optique fondationnaliste classique, on doit poser des principes ex ante, desquels on tire ensuite toutes les conséquences. Dans le cas de la perspective linéaire que nous allons examiner, l'édifice intellectuel a été construit empiriquement et les fondations ont été reconstruites ex post de manière à se conformer au mieux au système préalablement mis en œuvre. Le cas de la perspective linéaire se signale à notre attention par la quantité de débats qu'il a engendrés durant les Quattrocento et Cinquecento. Dans ces échanges, l'historien trouvera les matériaux pour repérer les lignes de partage entre les différentes options fondationnalistes et pour comprendre les arguments par lesquels certaines options se sont imposées.

\section{JALONS DE LA MATHÉMATISATION PERSPECTIVE}

L'application de notions géométriques à la perspective est ancienne. Tenons-nous au critère selon lequel la mathématisation est reconnaissable à l'existence de démonstrations explicites. 
1470. Piero della Francesca. - L'histoire de la mathématisation perspective débute dans le De prospectiva pingendi de Piero della Francesca (1470). ${ }^{5}$ On lui doit d'avoir explicité la diminution des grandeurs apparentes à partir des triangles semblables et des relations de proportionnalité. Voici la structure du De prospectiva pingendi, prop. I, xiii:

La figure associe le plan (le carré $B C F G$ à représenter), le profil (l'œil $A$ et le tableau $B F$ ), et la perspective $\left(B C D^{\prime} E^{\prime}\right)$. Le segment $B E$ est l'image de $B C$ (resp. $E H$ image de $C G ; H F$ image de $G F$ ), parce que $B E$ et $B C$ sont sous-tendus par le même angle $B A C$ (resp. $E A G ; G A F$ ). Cette construction exhibe les rapports $A E: A C=D B: D C, E H: C G=A E: A C$. Le rayon $A C$ interceptant le tableau en $E$, la perspective de $B C F G$ est $B C D^{\prime} E^{\prime}$, “dico avere quadrato il piano degradato." Sur le quadrilatère $B C D^{\prime} E^{\prime}$ on a les rapports $D^{\prime} E^{\prime}: B C$ $=A^{\prime} D^{\prime}: A^{\prime} B=A^{\prime} E^{\prime}: A^{\prime} C$. La prop. I, xiii s'achève par l'affirmation que les mêmes rapports existent, quelle que soit la position du point de fuite $A^{\prime}$ sur l'horizon, "nientedimeno se po mectare dove a l'omo piaci [...] et dove tu il mecterai verrà in quella medesima proportione."6 (Figure 3).

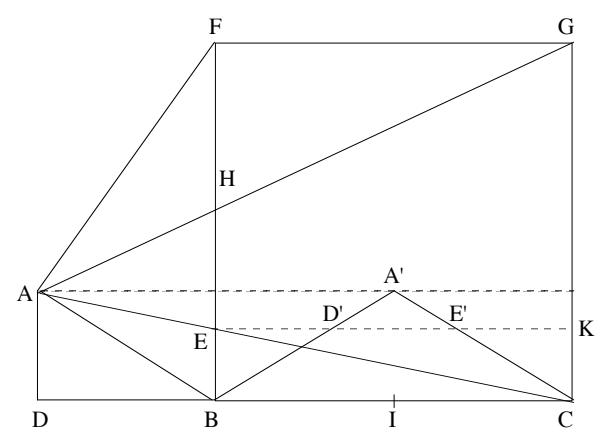

Figure 3

1583. Egnatio Danti. - Un deuxième jalon est la preuve d'existence du point de fuite pour un groupe de droites de bout, donnée par Danti dans son commentaire des Due Regole della prospettiva pratica de Vignola (1583), prop. xxxiii:

Soit $A B C D$ le carré à représenter, $C D E F$ le tableau, l'œil étant en $K$. Les points $C$ et $D$ de la ligne de terre ne sont pas modifiés. Les rayons visuels $K A$ et $K B$ interceptent le tableau en $G$ et $H$. Donc, le quadrilatère $C D G H$ est la perspective du carré $A B C D$. Les triangles $K I G$ et $G D A$ (resp. $K I H$ et $H C A$ ) sont équiangles. Donc $K I: I G=A D: D G$ et $K I: I H=B C: C H$ et, par permutation, $I G: G D=K I: D A$ et $I H: H C=K I: C B$. Comme $A D=B C$, on a $I G: G D=I H: H C$. Les côtés de $D I C$ étant coupés proportionnellement en $G$ et $H$, $G H$ et $D C$ sont parallèles. Dans $K A B, A K: G K=A B: G H$. Puisque $A K>G K$, alors $A B=C D>G H$. Donc, il existe un point de concours des droites $D G$ et $C H$. C'est le point de fuite $I$, projection orthogonale de l'oeil $K$ sur le tableau: $I O=K M$. La perspective de $A B C D$ est donc " $D G H C$, li cui due lati paralleli $D G$, et $\mathrm{CH}$, allungandosi vanno a terminare nel punto I dell'orizonte." (Figure 4). ${ }^{7}$

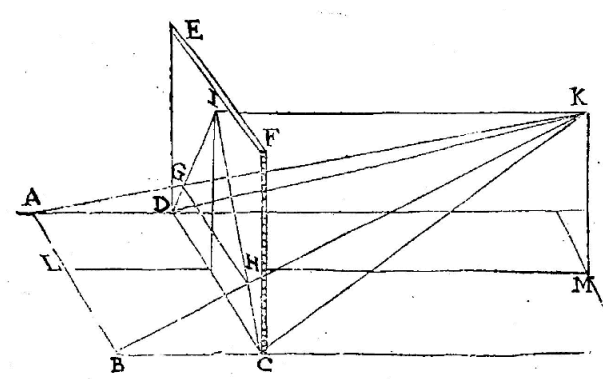

Figure 4 
1643. Girard Desargues. - Une troisième contribution majeure à la mathématisation perspective est celle que donne Desargues dans la "Première proposition géométrique," probablement publiée dans le Livret de perspective (1643), puis annexée à la Maniere vniverselle de Mr Desargves pour pratiquer la perspective de Bosse (1648):

Si les droites $D a, E b, K l$, de deux triangles homologues $D E K$, $a b l$ concourent au point $H$, centre de projection, les points d'intersection $c, f, g$ des droites homologues sont colinéaires (Figure 5). - Premier cas. Les triangles $D E K$ et $a b l$ sont dans des plans différents. Les points $c, f, g$, appartiennent au plan $D E K$, dans lequel sont les droites $D E c, D f K, E g K$, et au plan $a b l$, dans lequel sont les droites $a b c, b f l, a g l$. L'intersection des deux plans étant une droite, les points $c, f, g$, sont colinéaires. - Deuxième cas. Les triangles $D E K$ et $a b l$ sont dans le même plan. La démonstration de Desargues est concise, mais on y reconnaît le théorème de Ménélaüs. Appliqué aux couples $\{D H K, a l\},\{H E K, b l\},\{E D H, a c\}$, il donne les équations $g D: g K=a D: a H . l H: l K ; f K: f E=l K: l H . b H: b E ; a D: a H=c D: c E . b E: b H$, dont on tire $c D: c E=$ $g D: g K . f K: f E$. Sa réciproque sur $\{D E K, c f\}$ établit alors la colinéarité de $c, g, f$. -Application perspective. Attachons le triangle $D E K$ au plan de terre $\mathscr{G}$, le triangle $a b l$ au plan du tableau $\mathscr{T}$, l'œil étant en $H$. Les points $c, f, g$ définissent alors la ligne de terre. Si maintenant deux côtés des triangles homologues sont parallèles alors on retrouve, comme cas particulier, la méthode du tiers poinct présentée par Viator. ${ }^{8}$ L'intérêt du théorème tient au fait que "la figure [...] correspond droite à droite; point à point; et raison à raison; à celle de diuers plans, et l'on peut discourir de leurs proprietez sur l'vne comme sur l'autre, et par ce moyen se passer de celle du relief en luy substituant celle d'un seul plan"`9 (Figure 6).

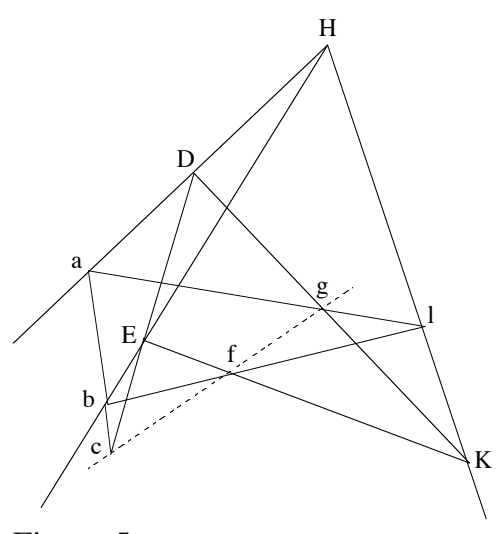

Figure 5

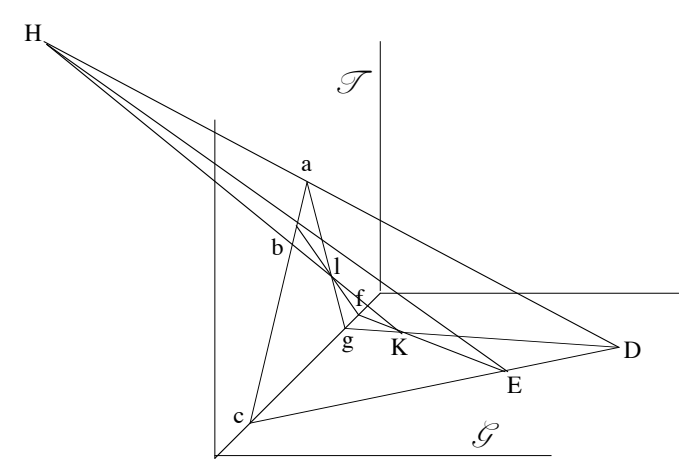

Figure 6

Usage du théorème de Thalès dans le plan par Piero della Francesca; emploi du théorème de Thalès sur des triangles opposés par le sommet dans l'espace par Egnatio Danti; recours au théorème d'incidence et au théorème de Ménélaüs par Girard Desargues: voilà quelques exemples d'application de la géométrie aux problèmes de perspective.

On observe d'emblée que le travail engagé par les architectes mathématiciens est irréductible à la présentation des traités en définitions, suppositions, théorèmes, lemmes et scholies. Ces effets de style euclidiens ne nous semblent pas prêter à conséquence. En revanche, l'histoire perspective constitue une activité de mathématisation en raison de son caractère démonstratif (quatrième composante). La volonté de substituer la certitude démonstrative à l'intuition a permis de poser et de résoudre de nouveaux problèmes (unicité du point de fuite, par exemple). 


\section{RÉSISTANCES À LA MATHÉMATISATION}

Jusqu'à la fin du Cinquecento, les tableaux italiens ont suivi une grande variété de principes constructifs. La stabilisation que l'on observe ensuite tient à la codification des règles de perspective en vue de son enseignement. C'est principalement au sein des académies qu'a eu lieu cette transformation, et c'est d'ailleurs pour des motifs didactiques qu'ont été composés les traités de perspective de Serlio (1545), Cataneo (1567), Barbaro (1569) ou Danti (1583).

En marge du processus de codification de la perspective linéaire, beaucoup de peintres continuaient de suivre des règles empiriques ou hétérodoxes (Vittore Carpaccio, La Présentation au Temple, La Naissance de la Vierge, ca. 1504). La résistance de ces alternatives est établie par les nombreux traités qui fustigent l'ignorance des praticiens et condamnent leur incurie. Dans La Pratica della perspettiva, Barbaro critique les artistes qui refusent d'admettre l'autorité des mathématiques sur leur pratique et se satisfont de procédés rudimentaires (semplice pratica). ${ }^{10}$ Dans les Due Regole, Danti attaque les peintres qui suivent de fausses règles en perspective. ${ }^{11}$ Les plaidoyers en faveur de la mathématisation firent face à l'opposition de tous ceux qui, pour diverses raisons (évidence visuelle, sens inné de la perspective, arguments physiologiques), excluaient qu'on puisse mathématiser la peinture. En 1607, on trouve encore un Federico Zuccaro pour écrire:

L'art de la peinture ne tire pas ses principes des sciences mathématiques, et il n'y a aucun besoin de recourir à elles pour apprendre les règles et les moyens de son art, ni même pour pouvoir raisonner spéculativement. En vérité, ce n'est pas d'elles qu'elle est la fille, mais bien de la nature et du dessin [...] Si l'on voulait examiner et connaître toutes les choses par spéculation mathématique [...], outre l'intolérable fatigue, ce serait une perte de temps sans aucun bénéfice substantiel. ${ }^{12}$

L'existence et l'âpreté de ces polémiques montrent la difficulté que les praticiens ont eu à discerner les fondements rationnels du système perspectif. Le siècle et demi de temps qui s'est écoulé entre les recherches présumées de Brunelleschi et le moment de la stabilisation académique s'explique par les obstacles rencontrés dans le choix de postulats compatibles avec le tracé des épures. Les progrès de la géométrisation perspective ont été subordonnés à la sélection d'hypothèses fonctionnelles, parfois hautement contre-intuitives . On peut clarifier ces choix, et les débats qui les ont accompagnés, en les ramenant à quatre alternatives.

\section{CHAMP DE VISION LARGE VS. LIMITÉ}

Les règles de la perspective linéaire trouvent une première limite naturelle dans le problème des déformations latérales. Plus la partie représentée est éloignée de l'axe de vision, plus les déformations latérales paraissent inacceptables. Il n'existe pas de manière optimale de résoudre le problème: soit la perspective embrasse un champ visuel étendu et produit ces déformations; 
soit elle élimine les distorsions, mais découpe alors un champ visuel limité peu conforme aux conditions de la vision naturelle. Certains peintres du Quattrocento y virent l'échec de la géométrisation de l'espace pictural. Piero della Francesca tenta de résoudre ce problème. Voici ce qu'il écrit dans le De prospectiva pingendi, prop. I, xxx:

Pour sortir de l'erreur ceux qui ne sont pas très experts en cette science et qui disent que [...] ils trouvent le raccourci plus grand que ce qui n'est pas en raccourci - ce qui arrive pour ne pas avoir compris quelle distance il doit y avoir de l'œil à l'écran où l'on place les choses [...] de sorte qu'ils doutent que la perspective soit une vraie science $[\ldots]$.

Je ferai donc le dessin d'un carré aux lignes égales et parallèles, soit $B C D E$ [construction de la figure, chaque œil, en $A$, voyant un des secteurs $B A C, C A E, E A D$ ou $D A B]$. Par l'intersection de deux petits nerfs qui se croisent, le pouvoir visuel vient au centre de l'humeur cristalline, et de celle-ci partent les rayons qui s'étendent en ligne droite, (1) découpant un quart du cercle de l'œil [...] (2) Mais si on dépassait la diagonale, l'autre œil serait moins que le quart du cercle, ce qui ne se peut pas parce que les diagonales du carré parfait divisent le cercle en quatre parties égales ; ainsi, $F G$ est l'étendue maximum qu'un tel œil puisse voir. Il en résulte donc qu'en dépassant cette limite, la longueur en raccourci est plus grande que celle qui n'est pas en raccourci parce qu'elle entre dans la zone de vision de l'autre œil. La preuve: que l'on joigne $B 12345678 C$ au point $A$. Je dis que la ligne $B$ sera la diagonale passant par $F$ de la ligne $F G$, et si tu ajoutes à la ligne $B C$ la longueur qui se trouve de 1 à $B$, à la suite, soit $B K$, et qu'à 21 tu joignes la longueur de $F$ à 21 , soit $21 L$, mène ensuite $K L$, faisant un carré qui est $B K L 21$; si l'on joint $K$ au point A on coupera $21 F$ au point $M$. (3) Je dis que $K L$, qui est en raccourci, est supérieur à $21 L$ non raccourci, de la longueur $21 M$, parce que $K L$ se représente égal à $L M$, qui est supérieur à $L 21$; comme j'ai dit que le raccourci est supérieur à ce qui n'est pas en raccourci, cela ne se peut pas, car l'œil ne peut pas dans ces limites voir $K$ qui est dans la zone de l'œil opposé à la ligne $F H$.

Et bien que l'œil voie $F G$, l'esprit ne conçoit pas et ne comprend pas ses bords sinon comme une tache vue de loin qu'il ne sait juger [...] Il est donc nécessaire de prendre un écran plus petit que la ligne $F G$; (4) pour que l'œil perçoive plus facilement les choses qui lui sont opposées, il faut qu'elles se présentent sous un angle inférieur à l'angle droit ; je dis qu'il est des deux tiers d'un angle droit, parce que les trois composent un triangle équilatéral, chaque angle étant forcément aussi grand que l'autre.

Je dis que, si ton travail a sept brasses de large, tu t'éloignes d'au moins six brasses pour regarder, et s'il était plus grand, que tu te tiennes dans les mêmes proportions ${ }^{13}$ (Figure 7).

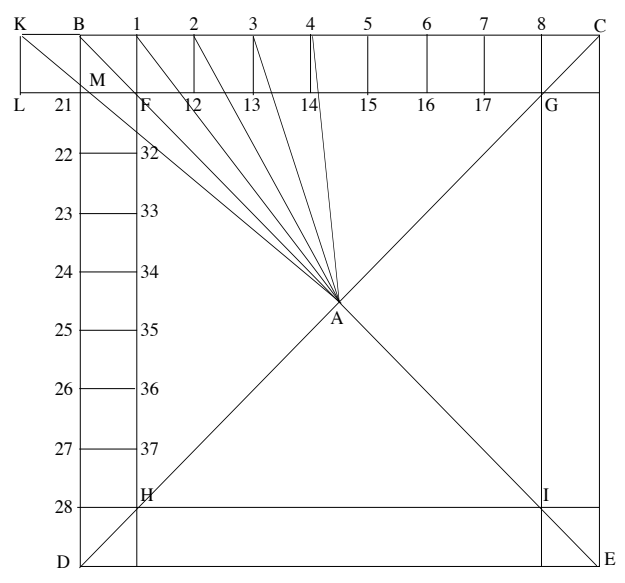

Figure 7

Voici la structure du raisonnement: 1) Piero prend acte des menaces qui pèsent sur le statut de science de la perspective: "alchuni [...] stanno in dubitatione la prospectiva non essere vera scientia." 2) Définition: il y a déformation latérale quand, dans un carré vu en perspective, un 
côté est plus grand que le côté en vraie grandeur."14 3) Le problème résulte du fait que les peintres ignorent la distance à laquelle on doit se tenir pour voir le tableau. 4) Piero construit la figure. 5) Le champ visuel doit découper un angle droit au centre de l'œil, sinon la perspective $L M$ apparaît plus grande que le côté en vraie grandeur $K L=L 21$. 6) Les extrémités du champ visuel étant vues confusément, il faut prendre une valeur inférieure à $90^{\circ}$. 7) Piero retient la valeur arbitraire $60^{\circ} .8$ ) Il conclut par un exemple numérique. Cette démonstration est en très dessous de la rigueur à laquelle Piero nous a habitué dans le reste du traité.

(1) Le champ visuel monoculaire est de $150^{\circ}$; le champ visuel binoculaire, obtenu par addition des deux champs monoculaires, s'étend sur plus de $180^{\circ}$; le champ visuel commun vaut $120^{\circ}$, mesures prises dans le plan horizontal. ${ }^{15}$ En retenant $90^{\circ}$, Piero hérite d'une valeur traditionnelle que Damianos ${ }^{16}$ attribue à Ptolémée. Cette valeur du champ de vision étant un lieu commun de l'optique médiévale, ${ }^{17}$ Piero n'avait pas de raison de la remettre en question. Par ailleurs, le De prospectiva pingendi (1475) est une pièce de l'optique pré-képlérienne. ${ }^{18}$ Or l'intervention de la rétine modifie la détermination du champ visuel. Dans l'optique postképlérienne, le rayon marginal le plus éloigné de l'axe de vision est celui qui pénètre dans l'œil latéralement pour atteindre le point de la rétine le plus excentré de la fovea (en dessous du corps ciliaire). Ce rayon fait un angle de $104^{\circ}$, ce qui fixe la valeur théorique ${ }^{19}$ du champ visuel à $208^{\circ}$. Dans l'optique pré-képlérienne, le rayon marginal le plus éloigné est le rayon rasant le bord de l'iris ${ }^{20}$ passant par le centre du cristallin. Il fait un angle d'environ $45^{\circ}$, origine de la valeur $90^{\circ}$.

(2) La démonstration est circulaire. Piero postule que le champ visuel est égal à un droit pour revenir au même résultat. En outre, le dispositif est trompeur. Piero suppose qu'un champ visuel "ampute" ou "pénètre" dans l'autre. Les champs visuels étant immatériels, ils peuvent être en intersection.

(3) Le passage dit en substance que la perspective de $L K$ est $L M>L 21$. Nous sommes ici au cœur de la démonstration et elle est fausse. Piero, qui raisonne en plan, se trompe en considérant que $L K$ se projette en $L M$. La droite $L K$ étant de bout, son image $L K^{\prime}$ passe par le point de fuite $O$, ce qui lui interdit d'être perpendiculaire à la ligne de terre $L G . L K^{\prime}$, perspective de $L K$, est l'hypothénuse du triangle $L M K^{\prime}$. On a donc toujours $L K^{\prime}>L M$ (Figure 8). Par ailleurs, en appliquant la définition des déformations latérales donnée par Piero à la vue perspective, on a montré que la valeur du champ visuel n'est pas $\angle F A G=90^{\circ}$, mais $\angle F A G=70^{\circ} 32^{\prime} .^{21}$ 


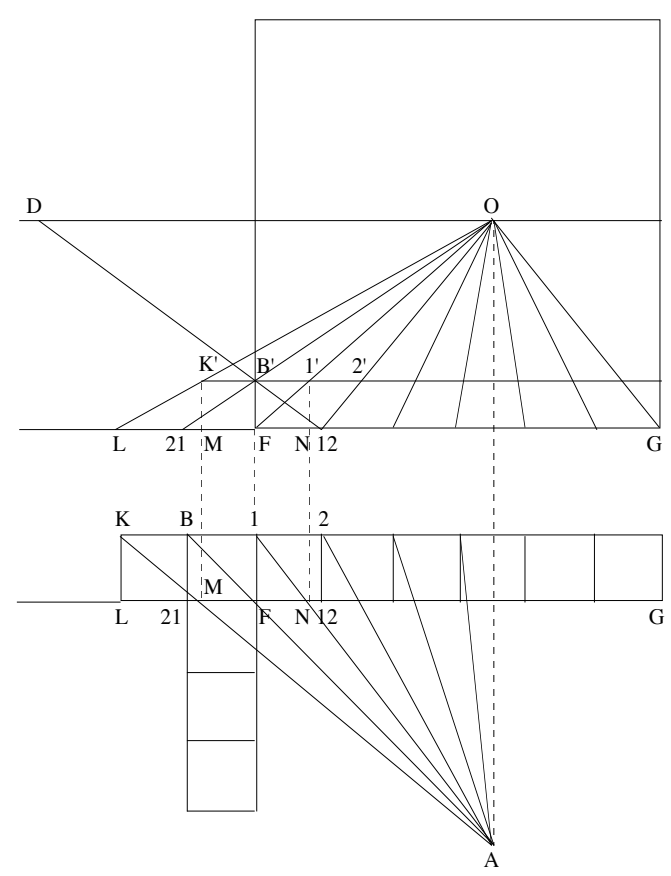

Figure 8

(4) L'œil voit indistinctement "se non commo una macchia" les objets périphériques du champ visuel, en particulier les points $F, G$ qui déterminent l'angle droit en $A$. Il propose alors de réduire l'angle de vision pour faciliter l'opération de la vue. Ce point fait naître un conflit entre la valeur du champ visuel qui a été démontrée $\left(90^{\circ}\right)$ et la valeur arbitraire qui sera finalement retenue $\left(60^{\circ}\right)$. Cette dernière n'est justifiée par aucune source historique. Les opticiens du Moyen-Âge, dont certains avaient envisagé l'existence d'un champ visuel inférieur à un droit, ${ }^{22}$ ne tentent pas d'en mesurer l'étendue: soit parce que la vision distincte est censée se produire quand les rayons perpendiculaires tombent à la surface du cristallin; soit parce qu'ils conçoivent une transition continue entre vision distincte et vision périphérique. ${ }^{23}$ Si Piero suit la première leçon, le champ de la vision distincte est identique au champ visuel total; il est donc inutile d'en restreindre la valeur à $60^{\circ}$. Si Piero suit la deuxième leçon, le problème n'est pas mathématisable: les déformations latérales sont simplement d'autant plus sensibles qu'on s'écarte de l'axe de vision.

Mais la prise en compte des déformations latérales n'est pas une nécessité. Ce n'est qu'une tolérance vis-à-vis de la mobilité de l'œil, examinée à la suite. La pseudo-démonstration de la prop. I, xxx, qui combine conformisme et arbitraire, sera largement suivie. Des œuvres d'une grande maturité, comme le commentaire de Danti, ${ }^{24}$ en hérite presque sans discussion.

\section{IMMOBILITÉ VS. MOBILITÉ OCULAIRE}


Dans la perspective, l'horizon et les points de fuite dépendent de la position du spectateur. L'horizon est à la hauteur des yeux; le point de fuite principal est l'intersection de l'axe de vision avec le plan du tableau. Si le spectateur modifie le regard, le point principal et l'horizon changent en conséquence. Lorber a bien vu qu'il y avait là un obstacle de la perspective. Il écrit: “À ce point, nous chercherons à comprendre la raison pour laquelle les traités de perspective ont réservé de plus en plus de place à l'anatomie de la vision [...] La réponse renvoie à une quaestio, toujours présente à l'histoire de la perspective, qui peut être résumée en ces termes: 1) la perspective est calculée pour un point de vue unique; 2) par sa nature même, le globe oculaire se meut constamment. ${ }^{, 25}$ Là réside la deuxième alternative de la perspective.

Dès le milieu du XVI ${ }^{\mathrm{e}}$ siècle, les théoriciens de la perspective se saisiront de la question. ${ }^{26}$ Il ressort de l'examen des débats que partisans et adversaires du rapprochement entre perspective et vision naturelle ont mobilisé des arguments spécieux pour fonder leur position.

En 1569, dans la Pratica della prospettiva, Barbaro reprend le problème de Piero della Francesca. Persuadé que le champ visuel est inférieur à l'angle droit, il soutient qu'un champ visuel plus étendu est une illusion psychologique due aux mouvements des yeux. ${ }^{27}$ Barbaro mobilise la rhétorique de la vérité et de l'erreur (inganna... quelli che non ricorreno alla ragione), de la certitude et de la croyance (è necessario... pare a gli imperiti altrimenti). En dépit de ces effets, l'argument est improbable: Barbaro confond champ visuel et "champ du regard. ${ }^{28}$ Or, comme il a été vu, le champ visuel normal est supérieur à $180^{\circ}$ en l'absence de tout mouvement.

Deux décennies plus tard, Danti reprend l'ancienne distinction entre aperçu (occhiata) et examen (sguardo) et montre que la perspective, qui relève exclusivement de l'aperçu, requiert la suspension du mouvement:

Il faut comprendre ici non pas ce que nous voyons simplement, mais ce que nous voyons d'un seuil coup d'œil, sans du tout bouger la tête, ni tourner l'œil. Ainsi (1) tout ce que représente la perspective, c'est ce que nous percevons en une ouverture de l'œil [...] Mais si l'œil n'était pas immobile et continuait à se mouvoir, il ne serait pas vrai que toutes les choses s'unissent en un seul point, étant donné que l'endroit où convergent toutes les parallèles de la perspective est face à l'œil. (2) S'il se déplaçait, le point [de fuite] se déplacerait aussi et entraînerait pareillement les lignes parallèles d'un point à l'autre, et l'on confondrait toutes les choses. Comme on le voit ici [sur la figure], si l'œil est en A, toutes les parallèles qui naissent aux points G, H, I, J, K et L vont s'unir au point C, duquel provient le rayon qui va au centre de l'œil A. [...] Si maintenant on déplace l'œil du point $\mathrm{A}$ au point $\mathrm{B}$, on déplacera également le point principal de la perspective du point $\mathrm{C}$ au point $\mathrm{D}$, dans lequel convergeront toutes les parallèles qui, précédemment, allaient au point C. Ainsi, en déplaçant l'œil, toutes les choses se transposent ${ }^{29}$ (Figure 9). 


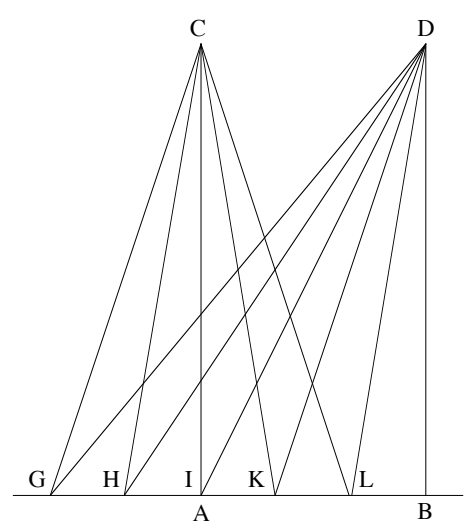

Figure 9

(1) La démonstration de Danti est basée sur la distinction entre "aperçu" (idrâk bi-albadîha, comprehensio superficialis; it. occhiata) et "examen” (idrâk bi-al-ta'ammul, comprehensio per intuitionem; it. sguardo). ${ }^{30}$ Nous savons aujourd'hui qu'il y a correspondance entre fonctions visuelles et structures anatomiques, du fait des deux systèmes bâtonnets-rétine périphérique-tectum vs. cônes-fovéa-système géniculo-strié. ${ }^{31}$ Ces deux systèmes ne sont pas antagonistes mais collaboratifs. Par conséquent, la perspective n'est pas un artefact relevant exclusivement de l'aperçu. Si le spectateur fait la fixation sur le point de fuite de la perspective, les conditions sont celles de l'examen pour une zone de $3^{\circ}$ à $5^{\circ}$ autour du point de fuite, celles de l'aperçu pour le reste du tableau. Les choses se présentent un peu différemment dans l'optique alhazénienne. La distinction entre les deux visions s'établit sur le fait que l'aperçu est un état, l'examen un processus. ${ }^{32}$ La fixation appelle donc l'aperçu, et le rapport que Danti établit entre perspective et occhiata est conforme à cette tradition.

(2) L'argument avancé par Danti ne dépend pas ici de l'optique physiologique mais de considérations relatives à la construction graphique des perspectives. Et c'est un argument difficilement opposable: on ne peut pas superposer des images d'une même scène ayant des points de fuite différents.

C'est donc essentiellement pour des raisons pratiques que le postulat de l'immobilité du regard s'est imposé. Les mouvements oculaires seront dès lors considérés comme des curiosités physiologiques sans incidence sur la pratique de la perspective.

\section{TABLEAU PLAN VS. CURVILIGNE}

Supposons une colonnade dans un plan frontal. Des angles égaux découpant des segments inégaux dans le plan du tableau, une perspective linéaire restitue des colonnes-objets de même diamètre par des colonnes-images de diamètre variable. Ce diamètre est d'autant plus grand que la colonne est éloignée du rayon central. Là se trouve la troisième alternative de la perspective. 
Dès que la scène représentée est assez large, l'œil est en mesure de comparer le diamètre des colonnes et d'apercevoir que les colonnes les plus larges sont les plus éloignées de l'axe de vision. La perspective curviligne résout ce problème: elle substitue au tableau plan une calotte sphérique centrée sur l'œil afin que les colonnes présentent le même diamètre. Les principes de la perspective curviligne ont été systématiquement étudiés à la fin du XIX siècle. Mais cette alternative à la perspective linéaire a des sources anciennes, dont on a voulu retrouver la trace chez Vinci. ${ }^{33}$

On dénombre une douzaine de notes de Vinci sur la perspective curviligne. ${ }^{34}$ Dans le Codex Atlanticus, fol. $1^{\text {ter }}$ (ca. 1508), Vinci écrit: "Fra lle chose d'equal grandeza, quella che sia piu distanta dall'ochio si dimossterra di minor figura." Le même énoncé se trouve dans le ms. E de l'Institut de France, fol. 4r (1513-1514), inséré cette fois dans un développement plus conséquent:

Problème de perspective. - [Savoir] si une longue paroi de 4 côtés parallèles et 4 angles droits présentera à l'œil des limites supérieures et inférieures rectilignes ou curvilignes. Des choses de même grandeur, la plus éloignée apparaitra d'autant plus petite qu'elle sera plus éloignée. Il s'ensuit que OP, largeur de la paroi RTOP, est plus petite que la largeur $\mathrm{AB}$ comme la ligne $\mathrm{PF}$ est plus longue que $\mathrm{BF}$, laquelle ligne $\mathrm{PF}$ dépasse BF de la longueur PM, qui est le tiers de l'espace PF. Donc, OP sera plus petit que AB d'autant, c'est-à-dire de $1 / 3$, et cette proportion sera soustraite, parce qu'une distance plus grande rend la chose plus petite, et une distance plus petite augmente la chose vue ${ }^{35}$ (Figure 10).

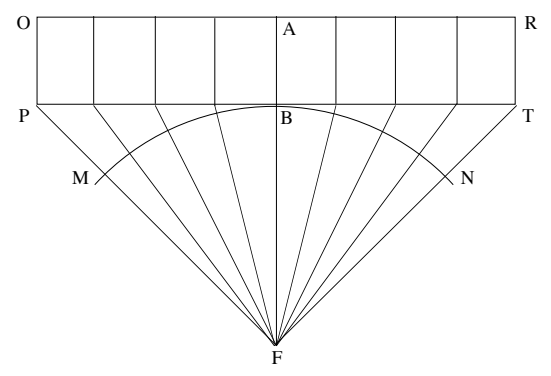

Figure 10

Vinci considère que l'œil perçoit inégaux les segments $O P<A B$. Mais on ne sait pas si cette observation a pour but de comparer la perspective naturelle et la perspective artificielle, ou si la remarque appelle un système de perspective curviligne. Ces notes ont donné lieu à des interprétations contradictoires, les uns soulignant que Vinci considère la perspective curviligne comme une "cosa più disputativa che da usarla." ${ }^{36}$ les autres arguant que Vinci parle d'une "prospettiua, fatta dall'arte sopra sito equalmente distante dall'ochio," ce qui implique bien l'usage du tableau curviligne. ${ }^{37}$

Dans son commentaire, Danti examine et réfute la possibilité de la perspective curviligne. Voici ce qu'il dit du perspectographe curviligne de Lanci:

Cet instrument [...] est ainsi fait. Sur un plateau rond, on fixe une tablette [...] comme le montre [la figure] HLKI, fixée au plateau rond GHSI. Au centre du plateau on fixe un axe, qui tourne autour du point A, et aux points $\mathrm{C}, \mathrm{B}$, on cloue la réglette $\mathrm{SE}$, de sorte que le clou pivote. Au sommet, on met une autre 
réglette avec deux mires à angle droit pour regarder de près ou de loin les choses qu'on veut mettre en perspective (Fig. 11). (1) Si l'on retire la feuille sur laquelle la perspective est dessinée pour la coucher sur un plan, toutes les choses deviennent fausses, et je le montre ainsi. Soient les grandeurs AF, FE, ED et DB, soit GIL l'instrument, et C l'œil par lequel, en regardant les points susdits, sont marqués au stylet les points de la feuille L, K, I, H, G. Maintenant, (2) si la feuille avec la perspective devait toujours rester fixée au cylindre, et regardée depuis le point $\mathrm{C}$, tout irait bien et les grandeurs, par exemple AF, et LK, étant vues sous le même angle $\mathrm{ACF}$, apparaîtraient égales, et se révèleraient identiques. (1) Mais comme la feuille est détachée de la circonférence LIG, et qu'on la couche sur le plan QOM, alors toutes les choses s'altèrent et se confondent. On verrait le point $\mathrm{A}$ en $\mathrm{Q}$ plutôt qu'en $\mathrm{S}[\ldots]$, et pareillement les autres points [F], D, B se verraient en dehors de leur place, aux points [P], N, $\mathrm{M}^{38}$ (Figure 12).

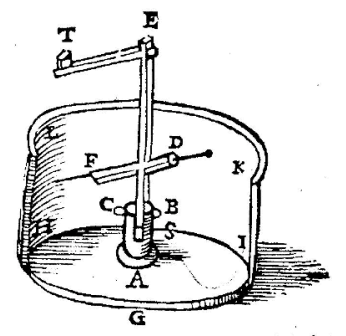

Figure 11

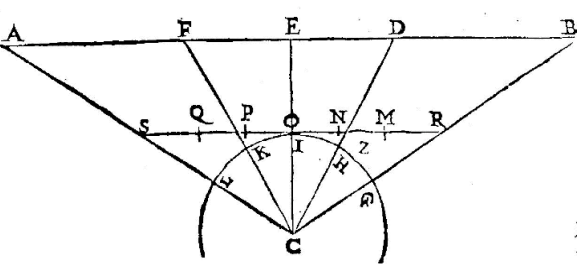

Figure 12

(1) Danti montre que la fausseté des perspectives réalisées à l'aide du perspectographe ne tient pas à son emploi, mais au développement de l'image curviligne sur un tableau plan. Il le démontre très simplement (Figure 12). Les grandeurs réelles $A F E D B$ sont prises sur le tableau curviligne $L K I H G$. Quand l'image est couchée sur le plan $S R, L$ correspond à $Q, K$ à $P, I$ à $O, H$ à $N, G$ à $M$. L'œil étant en $C$, les points-images $Q, P$, etc., ne sont plus sur les rayons $A C, F C$, etc. La perspective curviligne ne conserve donc pas les angles $\angle Q C P<\angle L C K$, etc.

(2) Danti est cependant conscient qu'une telle perspective demeure exempte de faute si elle reste attachée au tableau curviligne où elle a été tracée.

Les objections de Danti sont pour beaucoup dans l'éviction du système de perspective curviligne, qui n'a pratiquement jamais été discuté à l'époque classique. La perspective curviligne est, pour l'essentiel, une redécouverte du XIX ${ }^{\mathrm{e}}$ siècle.

\section{VISION MONOCULAIRE VS. BINOCULAIRE}

Une dernière alternative réside dans l'écart entre vision naturelle (binoculaire) et perspective artificielle (monoculaire). La perspective classique requiert le postulat de la vision monoculaire. Laurent rappelle que, dans la perspective linéaire, "les deux yeux de la vision binoculaire sont réduits en un seul (vision monoculaire) appelé œil et placé au sommet du cône visuel." ${ }^{39}$ Cette condition n'apparaît que rarement dans les textes anciens, par exemple dans le De visu Grazia de' Castellani: "E tu ponj un solo occhio al punto .c. doue è uno picholo bucho." textes sont épars, et il n'est pas rare de trouver des témoignages contraires. En réalité, le postulat de la vision monoculaire ne s'imposera définitivement qu'à la fin du Cinquecento. 
La rivalité entre les postulats de la vision binoculaire et de la vision monoculaire résulte de plusieurs facteurs. Le premier est évidemment le caractère naturel de la vision binoculaire ${ }^{41}$ qui suggérait d'en dériver un système perspectif. Le second facteur est que tous les traités d'optique issus de la tradition alhazénienne reconnaissent l'existence d'une vision binoculaire physiologique - non pathologique - incitation supplémentaire à fonder un système de représentation binoculaire. Certains perspectivistes se sont donc essayé à acclimater les principes d'optique physiologique que l'on trouvait dans les traités de perspectiva communis.

$1^{\circ}$ L'existence d'une alternative binoculaire de la représentation est attestée par la reconstruction ex post des épures perspectives et l'examen des tracés matériels. Une trentaine de peintures italiennes répondent à ces conditions. ${ }^{42}$ On les reconnaît essentiellement à deux "points de fuite principaux," qui ne doivent pas être confondus avec les points de fuite latéraux d'une perspective angulaire ou les points de distance d'une perspective centrale. Ces auvres présentent un dédoublement du point de fuite principal, chacun correspondant à chaque œil. Ce système de perspective hétérodoxe à deux points n'a pas totalement disparu à la Renaissance. En 1572, Bassi commente encore des œuvres à deux points principaux, comme le bas-relief de Tibaldi pour la cathédrale de Milan. ${ }^{43}$

$2^{\circ}$ Plusieurs textes d'époque témoignent des débats suscités par la perspective binoculaire. L'Architettura de Cataneo paraît en 1567. Le livre huitième, consacré à la perspective, contient une étrange réfutation du système binoculaire. Selon Cataneo, bien que nous ayons deux yeux, nous verrions plus distinctement d'un seul œil. Les borgnes auraient donc, en la matière, un avantage sur les bien-voyants. ${ }^{44}$ C'est un argument erroné. Le clignement d'un œil, que pratiquent les peintres et les architectes, n'est pas à l'origine de la vision nette, mais de la visée (la suppression d'une quasi-image neutralisant la rivalité rétinienne). ${ }^{45}$ De plus, la non correspondance exacte des points rétiniens assure la stéréopsie, qui est impliquée dans l'appréciation des distances et du relief.

Une réfutation plus conséquente du système binoculaire tombera en 1583, à la parution du commentaire de Vignola par Danti:

Il en est qui croient que, l'homme ayant deux yeux, [la perspective] doit s'achever en deux points [...] Celui qui a vu une anatomie de la tête a pu constater en même temps que (1) les deux nerfs optiques s'unissent de la même manière que la chose vue. Bien qu'elle entre par deux yeux, elle s'achève en un seul point du sens commun [...] Ce que nous voyons avec les deux yeux nous paraît unique et non double, car les pyramide qui se forment de la chose vue dans l'un et l'autre œil, qui sont comme les pyramides qui viennent aux deux yeux E, F, ont la même base, et (2) l'axe de l'une et de l'autre pyramide qui vont aux yeux, naissent du même point G. Les nerfs optiques, qui sont creux comme des pailles, s'unissent ensemble au point $\mathrm{H}$, où les espèces qui sont portées au sens commun par les esprits visuels, sont mélangées ensemble [...] Il s'ensuit que, avec deux yeux, on voit une seule chose, et qu'en perspective, il n'y a qu'un seul point ${ }^{46}$ (Figure 13). 


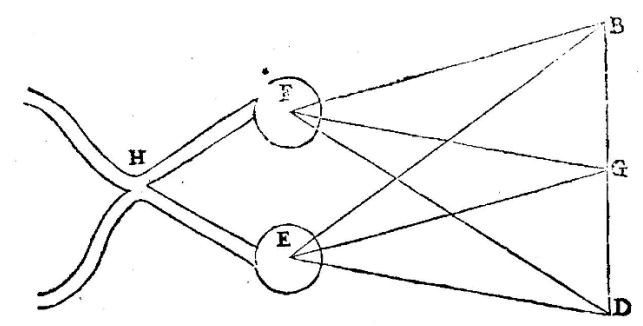

Figure 13

Danti mobilise un argument anatomophysiologique. L'entrecroisement des nerfs optiques au chiasma étant la marque même que le système visuel intègre les quasi-images issues des deux yeux, la vision est une. Il est donc vain de tracer des perspectives à deux points, "cosa absordissima, et contraria totalmente a quello che vediamo." Le passage soulève deux difficultés.

(1) Qu'il y ait croisement des nerfs optiques au chiasma ne signifie pas que la fusion des sensations visuelles s'opère par sommation. Nous savons aujourd'hui qu'il s'agit d'une intégration complexe, dans laquelle une disparité modérée des images est, non seulement tolérée, mais nécessaire à la perception du relief. Qu'en est-il dans les textes de la Renaissance ? Contre certaines lectures simplificatrices, on trouve d'indéniables éléments de modernité dans toute la tradition alhazénienne. ${ }^{47}$ En étudiant les conditions de fusion des quasi-images, Ibn al-Haytham a distingué l'existence de la diplopie physiologique. $1^{\circ} \mathrm{Si}$ les points-objets tombent en des points correspondants de l'oeil, il y a fusion simple: "Les deux formes qui apparaissent en deux points semblablement situés (fî nuqtatayni mutashâbihatay al-wad'/in duobus punctis... consimilis positionis) de la surface des yeux, viendront en un même lieu du nerf commun, où elles se superposeront et composeront une seule forme." $2^{\circ} \mathrm{Si}$ les points-objets tombent en des points disparates, ils sont vus doubles (yudraku 'ithnayni/comprehendetur duo). $3^{\circ} \mathrm{Si}$ les points-objets tombent en des points non correspondants, mais faiblement disparates, ils sont fusionnés. ${ }^{48}$ Au Moyen Âge, l'intégration des sensations visuelles tolère donc une disparité modérée. Si l'on revient au commentaire de Danti, on constate que les concepts de specie, spiriti visiui, senso commune attestent de sa dépendance à l'égard de l'optique médiévale. Il s'écarte pourtant de la tradition alhazénienne. Le passage "terminare in un sol punto nel senso commune" ne tient pas compte de l'étude de la fusion binoculaire faite par Ibn al-Haytham, qui ne décrit aucun isomorphisme entre chiasma et fusion des sensations visuelles.

(2) Si les bissectrices $E G$ et $F G$ des pyramides $B E D$ et $B F D$ tombent en un même point, $G$ est le point de fixation. On est surpris de voir Danti en faire un argument (perche/perciò) de l'unification des sensations visuelles. Cela ne peut se comprendre que rapporté à l'idée que le rayon axial de la pyramide est plus fort. Mais dans l'optique médiévale cette propriété est généralement stipulée du rayon perpendiculaire à la surface du corps. ${ }^{49}$ Si Danti se place dans le 
sillage de l'optique médiévale, les rayons perpendiculaires tombent en deux points, $G^{\prime}$ et $G^{\prime \prime}$, distincts de $G$, et ne contribuent pas à la fusion des quasi-images. Si Danti s'émancipe de cette tradition, alors les rayons $E G, F G$ sont quelconques et ne jouent plus aucun rôle particulier.

Les arguments avancés par Cataneo et Danti, quoique non entièrement convaincants, seront largement suivis par les praticiens et les théoriciens de l'époque classique, si bien que l'étude de la perspective binoculaire sera complètement abandonnée. La perspective à deux points, déjà perçue comme système hétérodoxe à la Renaissance, devient alors un système fossile. Beatus sit monoculus in terra binoculorum!

\section{CONCLUSION}

Les discussions engagées, du Quattrocento à la fin du Cinquecento, sur les fondements de la perspective linéaire ont abouti à la stabilisation de quatre postulats, qui sont autant de conditions préalables de la pratique perspective. Ces débats permettent de tirer plusieurs conclusions générales quant au processus de mathématisation.

Les progrès de la perspective ont souvent été accomplis par des hommes possédant une double compétence. L'histoire de la mathématisation perspective ne se résume pas dans un mouvement d'abstraction vis-à-vis de la pratique empirique des peintres et des architectes qui se serait produit, pour l'essentiel, à la charnière des $\mathrm{XVI}^{\mathrm{e}}$ et $\mathrm{XVII}^{\mathrm{e}}$ siècles $^{50}$.

1) La moindre division du travail qui régnait à l'époque fut une condition favorable du développement des «mathématiques pratiques ». Le progrès de la mathématisation perspective ne fut pas le fait exclusif de mathématiciens purs. Beaucoup de ceux qui écrivirent cette histoire avaient un profil mixte. En atteste la biographie des auteurs qui ont pris part aux débats étudiés dans cet article. Piero della Francesca (ca. 1420-1492) a exercé comme peintre et mathématicien; Giacomo Barozzi da Vignola (1507-1573) comme architecte; Baldassare Lanci (ca. 1510-1571) comme architecte et ingénieur militaire; Pietro Cataneo (ca. 1510-1574) comme architecte et ingénieur militaire; Guidobaldo del Monte (1547-1607) comme mathématicien et ingénieur militaire; Girard Desargues (1591-1661), comme architecte et mathématicien. Il reste quelques profils de non praticiens, dont l'intérêt pour les applications pratiques semble avoir résulté de circonstances familiales ou sociales: Federico Commandino (1509-1573) était fils d'architecte, Daniele Barbaro (1513-1570) était un ami de Palladio et de Veronese, le frère d'Egnatio Danti (1536-1586) était sculpteur.

2) L'idéologie mathématique - en particulier celle qui était cultivée dans les cercles patriciens vénitiens du Cinquecento - fut un autre facteur d'essor de la mathématisation de la perspective. ${ }^{51}$ On assiste en effet, d'abord à Venise, puis dans le nord de l'Italie et en France, à une forme de «pan-mathématisme », selon lequeltoutes les pratiques demandent à être fondées 
en raison et appuyées sur le raisonnement mathématique. On assiste alors à de multiples tentatives, plus ou moins coordonnées, qui auront pour résultat une extension du domaine des mathématiques à toutes ses applications. En 1634, le Cursus mathematicus d'Hérigone contient, outre les parties proprement mathématiques, l'arithmétique pratique, le calendrier (t. 2), la géométrie pratique, les fortifications, la milice, la mécanique (t. 3), la cosmographie, la géographie, la navigation (t. 4), l'optique, la catoptrique, la dioptrique, la perspective, la trigonométrie sphérique, la théorie planétaire, la gnomonique et la musique (t. 5).

3) Versés ou non dans une activité pratique, tous ces auteurs poursuivaient le dessein de faciliter le travail des praticiens, assez souvent en rapport avec les besoins de l'enseignement académique. Cette volonté d'épauler les praticiens apparaît dans l'intitulé même des textes de l'époque. ${ }^{52}$ Girard Desargues témoigne lui aussi de cette préoccupation :

J'avoue franchement que ie n'eus jamois de goust à l'estude ou recherche, ny de la phisique, ny de la géométrie, sinon en tant qu'elles peuuent seruir à l'esprit $[\ldots]$ en leur application pour la pratique de quelque art, m'estant aperceu, qu'vne bonne partie d'entre les pratiques des arts, est fondée en la géométrie ainsi qu'en vne baze assurée; entre autre celle de la coupe des pierres en l'architecture, estant pour cela nommées pratique du trait geométric; celle des cadrans au soleil [...]; celle de la perspectiue en l'art de la pourtraicture [...] Je m'aperceut que ceux qui s'y adonnent, auoient à se charger la mémoire d'vn grand nombre de leçons diuerses [qui] produisoient vn embarras incroyable en leur entendement [...], leur faisoient perdre du temps, surtout en celle de la pourtraicture, si belle et si estimable entre les inuentions de l'esprit humain, où la plus part des peintres et autres ouuriers traualloient, comme à l'aduenture et en tastonnant: sans guide ou conduite assurée, et par conséquent auec vne incertitude et fatigue inimaginable. Le désir et l'affection de les soulager [...] me fit chercher et publier des règles abregées de chacun de ces arts. $^{53}$

Ce témoignage interroge le pouvoir explicatif de la théorie de l'hybridation des rôles de Joseph Ben David. ${ }^{54}$ Cette théorie suppose l'existence de rôles sociaux définis et de pratiques scientifiques institutionnelles, ce qui est le cas de la science moderne. Par contraste, ni Piero della Francesca, ni Vinci, ni Desargues ne peuvent être considérés comme des transfuges d'une discipline-source vers une discipline-but. La théorie de l'hybridation est donc ici inapplicable. Il est cependant aisé de remonter à la racine commune de la théorie partielle de Ben David et du cas de la perspective linéaire. L'une et l'autre se ramènent à l'acquisition d'une double compétence. Ce concept exprime une condition plus générale d'apparition d'une innovation scientifique.

L'étude des quatre alternatives relève d'une discussion sur les fondements, dans laquelle on perçoit un travail d'axiomatisation faible. Les progrès de la mathématisation perspective offrent un profil très différent de la glose de Tûsî. Reprenons l'examen de la mathématisation comme axiomatisation, formalisation, quantification, introduction de concepts mathématiques ou 
caractère démonstratif. - Le De prospectiva pingendi de Piero, le commentaire de Danti ou la "Proposition géométrique" de Desargues ont un caractère démonstratif évident, souvent inspiré du paradigme euclidien. Du fait de l'applicabilité de la géométrie aux problèmes de la vision, ${ }^{55}$ la mathématisation de la perspective a manifestement profité du modèle d'une tierce-discipline. C'est l'optique géométrique qui a fixé l'horizon du possible. ${ }^{56}$ - La formalisation n'est pratiquement pas intervenue dans les progrès de la perspective, en tant que science géométrique. $^{57}$ - La quantification parâ̂t y avoir tenu un rôle subalterne. Le théorème des triangles semblables, utilisé par Piero puis Danti, le théorème de Ménélaüs utilisé par Desargues consistent en des rapports de grandeurs i.e. de quantités continues. Une certaine quantification a donc été présupposée par la mathématisation de la perspective. Mais elle a été importée avec la géométrie. Aucun travail spécifique de quantification n'a été engagé à cette occasion. ${ }^{58}-\mathrm{La}$ dimension axiomatique du travail est plus nettement perceptible dans les débats sur les fondements de la perspective. Revenons à l'architecture euclidienne. Classiquement, depuis Aristote, les principes liminaires se divisent en "axiomes" ou "notions communes," "définitions," "postulats" ou "demandes." Les solutions des alternatives ne concernent pas la signification des concepts: ce ne sont pas des définitions. N'ayant pas un caractère nécessaire, ce ne sont pas des axiomes. Étant sujettes à des choix opposables, elles constituent des postulats. ${ }^{59}$ Mais le travail engagé au XVI ${ }^{\mathrm{e}}$ siècle témoigne d'une axiomatisation faible, qui n'a pas été poussée à son terme. Même dans des textes éclairés, comme celui de Danti, on ne trouve pas toujours les postulats à leur place.

Au contraire du paradigme euclidien, l'axiomatisation faible engagée sur les fondements de la perspective a résulté d'un travail a posteriori. Au contraire d'Euclide, qui a procédé par voie déductive, des axiomes, définitions et postulats vers les théorèmes, l'élucidation des fondements de la perspective a été conduite à rebours. Alberti ${ }^{60}$ a exposé l'intersection de la pyramide visuelle sans s'interroger sur les préalables de cette géométrisation. Cela aurait dû constituer une "réflexion liminaire" de la perspective linéaire ; ce ne fut pas lecas. Le sentiment de certitude démonstrative émanant du modèle de l'optique géométrique a probablement occulté les difficultés que risquait de poser la mathématisation de la perspective. De là, le retour sur les fondements qui caractérise le Cinquecento. De là, le caractère codificateur et défensif du mouvement de mathématisation: il s'agissait à la fois de poser les bases d'un enseignement académique cohérent et de défendre le système de la perspective linéaire contre les attaques combinées de l'empirisme (l'expérience des peintres), de l'innéisme (la perspective comme sens inné) et de la physiologie (mobilité oculaire, vision binoculaire, notamment).

\footnotetext{
*Université Pierre-Mendès-France, BP 47, 38040 Grenoble Cedex 9, dominique.raynaud@upmf-grenoble.fr.
} 
${ }^{1}$ Euclide, Éléments, I, 1, ed. Bernard Vitrac (Paris, 1990), 194-197; Nasîn al-Dîn al-Tûsî, Tahrîr Kitâb al-Usûl li-Uqlîdis, cité par Khalid Bouzoubaâ Fennane, "Réflexions sur le principe de continuité," Arabic Sciences and Philosophy, 13 (2003), 101-136, cit. 109. L'attribution à Tûsî est contestée.

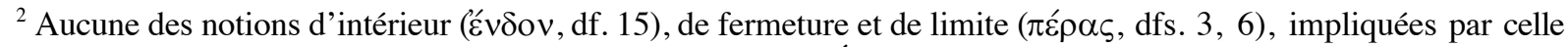
de frontière (ǒpos, df. 13), ne sont définies par Euclide, cf. Éléments, 153, 161-162.

${ }^{3}$ Par exemple, Le Hasard du nombre $\pi$ de François Morellet.

4 "Connaître scientifiquement, c'est savoir par démonstration," Aristote, Seconds Analytiques, ed. Pierre Pellegrin (Paris, 2005), 67-68.

${ }^{5}$ Le rôle joué par Piero della Francesca est mis en relief par Judith Field, "Alberti, the abacus and Piero della Francesca's proof of perspective," Renaissance Studies, 11 (1997), 61-88; Martin Kemp, "Geometrical perspective from Brunelleschi to Desargues," Proceedings of the British Academy, 70 (1984), 89-132.

${ }^{6}$ Piero della Francesca, De prospectiva pingendi, ed. G. Nicco Fasola (Firenze, 1984), 76-77.

${ }^{7}$ Egnatio Danti, Les Deux Règles de la perspective pratique de Vignole (1583), trad. Pascal Dubourg Glatigny (Paris, 2003), 192-193. Guidobaldo del Monte, Perspectiva libri sex (Pesaro, 1600), généralisera ce résultat, en l'établissant pour n'importe quel groupe de parallèles d'un plan horizontal.

${ }^{8}$ Jean Pélerin Viator, De Artificiali perspectiva (Toul, 1505), 2v; Lilyane Brion Guerry, Jean Pélerin Viator. Sa place dans l'histoire de la perspective (Paris, 1962), 142.

${ }^{9}$ Girard Desargues, "Première proposition géométrique," Euvres de Desargues, éd. Noël-Germinal Poudra (Paris, 1864), 1, 413-415; René Taton, L'Euvre mathématique de Girard Desargues (Paris, 1951); Judith V. Field and Jeremy J. Gray, The Geometrical Work of Girard Desargues (New York, 1987). La figure 6 est adaptée de Kemp, "Geometrical perspective," 126. Le théorème de Desargues est un théorème de colinéarité comme les théorèmes de Ménélaüs et de Pappus, avec lesquels il a des affinités évidentes.

${ }^{10}$ Daniele Barbaro, La Pratica della perspettiva (Venezia, 1569), proemio.

${ }^{11}$ Danti, Due Regole, 285, 287.

${ }^{12}$ Federico Zuccaro, L'Idea de' scultori, pittori e archittetti (Torino, 1607) in Trattati d'arte del Cinquecento, ed. Paola Barocchi (Bari, 1962), 1, 1043.

${ }^{13}$ Nous reproduisons, à quelques détails près, le texte établi par Marie-France Clergeau, Piero della Francesca, De prospectiva pingendi, thèse, Université Paris IV, 1987, 83-88.

14 “Piero chooses to define 'distorsion' (i.e. unacceptable distorsion) as occuring when the perspective renderings of tiles edges come out longer than the original ('perfect') tile edge. The definition as arbitrary, as is the rule with mathematical definitions, but it is in no way unreasonable," Judith Field, "Piero della Francesca's treatment of edge distorsion," Journal of the Warburg and Courtauld Institutes, 49 (1986), 66-99, cit. 69.

${ }^{15}$ Henry Saraux et Bertrand Biais, Physiologie oculaire (Paris, 1983), 242. Le champ monoculaire théorique est encore plus grand: il est évalué à $208^{\circ}$ par Yves Le Grand, Les Yeux et la Vision (Paris, 1960). C'est probablement par inadvertance que certains historiens affirment que la valeur actuelle du champ visuel monoculaire serait comprise entre $90^{\circ}$ et $115^{\circ}$, Thomas Frangenberg, "The image and the moving eye," Journal of the Warburg and Courtauld Institutes, 49 (1986), 150-171, cit. 159.

${ }^{16}$ Damianos Schrift über Optik mit Auszügen aus Geminos, ed. Richard Schöne (Berlin, 1897), 5; Maurizio Lorber, "Magia naturalis," La Prospettiva, ed. Rocco Sinisgalli (Firenze, 1998), 233-245, cit. 240, n.11. Ptolémée aurait dû traiter ce problème en II, 47-48, L'Optique de Claude Ptolémée, ed. Albert Lejeune (Leiden, 1989).

${ }^{17}$ The 'Opus majus' of Roger Bacon, ed. John Bridges (Frankfurt a.M., 1964), 59; John Pecham and the Science of Optics, ed. David Lindberg (Madison, 1970), 123.

18 "Je dis que la vision se produit quand l'image [...] se forme à la surface de la paroi concave de la rétine," Johannes Kepler, Ad Vitellionem paralipomena (Frankfurt a.M., 1604), Johannes Kepler Gesammelte Werke, ed. Max Caspar (Munich, 1938), 2, 168; Judith Field, “Two mathematical inventions in Kepler's Ad Vitellionem paralipomena," Studies in History and Philosophy of Science, 18 (1987), 1-19. La contribution de Kepler ne doit pas être surestimée. Plusieurs savants avaient reconnu le rôle de la rétine. André Vésale, De humani corporis fabrica (Bâle, 1543), 324, rapporte que beaucoup déjà la considèrent comme l'organe principal de la vision. Felix Platter, De corporis humani structura et usu (Bâle, 1583), 187, maintient que le nerf optique se déploie sur tout l'hémisphère rétinien pour recevoir les images des objets extérieurs. La description complète de l'œil, assimilant le cristallin à une lentille projetant l'image visuelle sur la rétine, est due à Christoph Scheiner, Oculus, hoc est fundamentum opticum (Inspruck, 1619). 
${ }^{19}$ Le champ visuel monoculaire est inférieur à cette valeur théorique car le rayon lumineux doit tomber sur une rétine fonctionnelle, or la distribution des cellules rétiniennes décroît de la fovea vers la périphérie.

${ }^{20}$ En réalité, la détermination du champ visuel se complique d'un problème supplémentaire dans l'optique préképlérienne, du fait de l'ouverture pupillaire variable (myosis, mydriase).

${ }^{21}$ Field, "Piero's treatment of edge distorsion," 71, 77-79.

22 "Igitur si in centro uuee esset uisio uideretur sub angulo recto precise si dyameter foraminis esset latus quadrati precise. Nunc autem centrum oculi, quod est anterioris glacialis, interius est quam centrum uuee quia uuea est minor quam cornea, et secat corneam quia foramen eius cornee applicatur. Igitur maximus angulus sub quo est uisio radiosa est minor recto nisi foramen uuee sit paulo maior quantitate predicta," John Pecham, 122.

${ }^{23}$ Thèse 1: "Visio distincta fit solum secundum perpendiculares lineas a punctis rei uisa ad oculi superficiem productas," Optica Thesaurus [...] Vitellonis Thuringopoloni libri decem, ed. David Lindberg (New York, 1972), 92. Thèse 2: "Unde sola perpendicularis illa que axis dicitur, que non frangitur, rem efficaciter representat, et alii etiam radii quo ei sunt propinquiores eo fortiores et potentiores in representando," John Pecham, 120.

24 "Et questo cono quando vediamo distintamente et perfettamente, è d'angolo acuto uguale all'angolo del triangolo equilaterale," Danti, Due Regole, 142.

${ }^{25}$ Lorber, "Magia naturalis," 234.

${ }^{26}$ Frangenberg, "The image and the moving eye."

27 "Necessario è che non si possa vedere sotto l'angulo largo. Et se pare a gli imperiti altrimenti, questo prociede dal velocissimo mouimento dell'occhio, il quale tracorre da uno termine all'altro per la mobilità sua, et inganna quelli, i quali non ricorreno alla ragione," Barbaro, La pratica della perspettiva, 8.

${ }^{28}$ Helmholtz, Optique physiologique, 2, 609; Saraux et Biais, Physiologie oculaire, 241.

${ }^{29}$ Danti, Due Regole, 222.

30 "Visio fit aspectu, aut obtutu [...] Comprehensio uisibilium erit secundum duos modos, qui sunt comprehensio superficialis, et comprehensio per intuitionem, quae profundum aspicit," Opticae Thesaurus Alhazeni, 67; The Optics of Ibn al-Haytham, Books I-III, ed. Abdelhamid Sabra (London, 1989), 1, $208-209$. Alhazen ajoute, Ibid., 209, que la vision soutenue est certifiée, contrairement à la vision d'ensemble. La théorie de la perception est exposée Ibid., 1, 126-138, 2, 78-79. Rappelons que la vision soutenue (al-ta'ammul, altafaqqud, comprehensio per intuitionem) n'est pas associée à la vision distincte (tafarruq, distincta visio), qu'Alhazen explique par une propriété constante de l'œil: l'arrangement des rayons qui tombent perpendiculairement à la surface du cristallin (al-rutûba al-jalîdiyya, humor glacialis sive cristallinus), Ibid., 1, 72-75.

${ }^{31}$ Saraux et Biais, Physiologie oculaire, 254-255; Renée Pigassou-Albouy, Les Strabismes, 2. Les convergences oculaires (Paris, 1992), 19-20.

32 "Obtutus fit in tempore. Et etiam comprehensio per intuitionem non erit, nisi in tempore: quoniam intuitio non erit nisi per distinctionem et motum uisus," Opticae Thesaurus Alhazeni, 71; Optics, I, 216.

${ }^{33}$ Carlo Pedretti, "Leonardo on curvilinear perspective," Bibliothèque d'Humanisme et Renaissance, 25 (1963), 69-87.

${ }^{34}$ Ms. A, fol. 38r; ms. E, fol. 4r et $16 \mathrm{r}$; ms. G, fol. $13 \mathrm{r}, 13 \mathrm{v}$ et $32 \mathrm{r}$; ms. $\mathrm{H}^{1}$, fol. $32 \mathrm{r}$; ms. K, fol. 40v et $41 \mathrm{r}$; Madrid II, fol. 15v; Br. M., Arundel 263, fol. 62r; Cod. Atl., fol. 35ra, The Notebooks of Leonardo da Vinci, 2 vols., ed. Jean Paul Richter (New York, 1972) passim.

${ }^{35}$ Pedretti, "Leonardo on curvilinear perspective," 85.

${ }^{36}$ John Elkins, "Did Leonardo develop a theory of curvilinear perspective?" Journal of the Warburg and Courtauld Institutes, 51 (1988), 190-196.

37 John White, The Birth and Rebirth of Pictorial Space (London, 1957), 207-215; Pedretti, "Leonardo on curvilinear perspective," 78. Le meilleur argument se trouve dans le ms. G, fol. 13v: "La semplice prospettiua è quella che è fatta dall'arte sopra sito equalmente distante dall'ochio con ogni sua parte - prospettiua conposta è quella che è fatta sopra sito il quale con nessuna sua parte è equalmente distante dall'ochio," Notebooks, I, 56.

${ }^{38}$ Danti, Due Regole, 238-240.

${ }^{39}$ Roger Laurent, La place de J.-H. Lambert (1728-1777) dans l'histoire de la perspective (Paris, 1987), 37.

${ }^{40}$ Gino Arrighi, "Un estratto dal 'De visu' di M Grazia de' Castellani," Atti della Fondazione Giorgio Ronchi, 22 (1967), 44-58, cit. 47; Filippo Camerota, "Misurare per perspectiva: geometria pratica e prospectiva pingendi," La Prospettiva, 293-308. Voir aussi The Life of Brunelleschi, by Antonio di Tuccio Manetti, ed. H. Saalman (University Park, 1970), 43. 
${ }^{41}$ Helmholtz, Optique physiologique, 2, 877-964; Yves Le Grand, Optique physiologique (Paris, 1956), 3, 201239; Charles Thomas, "Physiologie de la vision binoculaire," Archives d'Ophtalmologie, 31 (1971), 189-206; Saraux et Biais, Physiologie oculaire, 389-401. Helmholtz aborde le thème dans sa conférence de Berlin, Ernst Brücke et Hermann von Helmholtz, Principes scientifiques des Beaux-Arts, suivis de L'Optique et la Peinture (Paris, 1881), 174.

${ }^{42}$ Dominique Raynaud, "Une application méconnue des principes de la vision binoculaire: Ibn al-Haytham et les peintres du Trecento (1295-1450)," Oriens/Occidens, 5 (2004), 93-131; Id., "La théorie des erreurs et son application à la reconstruction des tracés perspectifs," in Marisa Dalai Emiliani et al., eds., L'Artiste et l'CEuvre à l'épreuve de la perspective (Rome, 2006), 411-430.

${ }^{43}$ Martino Bassi, Dispareri in materia d'architettura et perspettiva (Brescia, 1572). Pascal Dubourg Glatigny fait observer qu'il est "fait ici allusion à deux points principaux placés sur la même ligne d'horizon. Ceci doit être de pratique courante au XVI ${ }^{\mathrm{e}}$ siècle et ne suscite pas nécessairement l'indignation." Bassi réfute toutefois les constructions à deux points principaux en s'appuyant sur Euclide et Witelo, Danti, Due Regole, 447.

44 "Se nel fissar la vista in qual si voglia cosa o parte sua si serrerà un occhio, si giudicherà meglio che si tenessero ambidui aperti, però che la virtù d'ambedui gli occhi si riduce tutta in uno, e di qui è che quelli che hanno solo un occhio buono e dell'altro son ciechi veggano molto meglio da quel medesimo occhio che quelli che n'hanno due buoni," Pietro Cataneo, Dell'Architettura (Venezia, 1567), VIII, 16, Danti, Due Regole, 446.

${ }^{45}$ La rivalité rétinienne se produit en cas de forte disparité, Saraux et Biais, Physiologie oculaire, 394.

${ }^{46}$ Danti, Due Regole, 223-227.

${ }^{47}$ Opticae Thesaurus Alhazeni, 76-87; The 'Opus majus' of Roger Bacon, 92-99; John Pecham, 116-118; Optica Thesaurus Vitellonis, 98-108. À la suite des travaux d'Albert Lejeune, "Les recherches de Ptolémée sur la vision binoculaire," Janus, 47 (1958), 79-86, on a parfois confondu diplopie physiologique et strabisme pathologique, annulant ainsi toute possibilité de perception du relief: Gérard Simon, Le Regard, l'être et l'apparence dans l'optique de l'Antiquité (Paris, 1988), 131-132. Sur ce point, cf. Abdelhamid I. Sabra, Optics, 1, p. 228-243, 2, 111-127; Dominique Raynaud, "Ibn al-Haytham sur la vision binoculaire: précurseur de l'optique physiologique," Arabic Sciences and Philosophy, 13 (2003), 79-99.

${ }^{48}$ Opticae Thesaurus Alhazeni, 82; Optics, 1, 239 [28] (1 ${ }^{\mathrm{er}}$ cas), 234 [29] (2 $2^{\mathrm{e}}$ cas), 240 [33] (3 cas); Raynaud, "Ibn al-Haytham sur la vision binoculaire," 96-97.

49 "Quamuis enim a portione aliqua corporis spherici objecta rei patienti ueniat una pyramis habens radios infinitos [...] et omnes concurrunt in conum pyramidis cum radio perpendiculari, tamen unus solus est perpendicularis in una pyramide [...] et ideo est fortior, et plus habet de uirtute," The 'Opus majus' of Roger Bacon, 126-127.

${ }^{50}$ Ce mouvement d'abstraction progressive est très bien décrit par Kirsti Andersen, The Geometry of an Art. The History of the Mathematical Theory of Perspective from Alberti to Monge (New York, 2007).

${ }^{51}$ Palladio (1508-1580), Barbaro (1513-1570), Contarini (1536-1595), Barozzi (1537-1604) visaient à refonder l'architecture sur les mathématiques savantes - une attitude reprise hors du contexte vénitien au siècle suivant par Guarino Guarini (1624-1683), François Blondel (1618-1686) ou Philippe de la Hire (1640-1718). Paul L. Rose, The Italian Renaissance of Mathematics (Genève, 1975), Id., "Jacomo Contarini (1537-1595). A Venetian patron and collector of mathematical instruments and books," Physis, 18 (1976), 117-130.

${ }^{52}$ En attestent par exemple la Quinta parte del general trattato (Venise, 1560) que Tartaglia sous-titre: « Materia non men' utile che necessaria à Geometrici, Designatori, Perspettivi, Architettori, Ingegneri, \& Machinatori, si naturali, come Mathematici » ou la Geometria prattica de Pomodoro (Rome, 1599) elle aussi sous-titrée: «Opera necessaria à Misuratori, ad Architetti, à Geografi, à Cosmografi, à Bombardieri, à Ingegneri, à Soldati, \& à Capitani d'Eserciti ».

${ }^{53}$ Euvres de Desargues, 1, 24-25. Ce témoignage intervient directement dans la controverse sur les rapports entre géométrie projective et perspective. Il renforce la thèse de la continuité (Bkouche) contre la thèse de l'autonomie relative (Andersen) ou complète (Elkins) des deux disciplines, Rudolf Bkouche, "La naissance du projectif: de la perspective à la géométrie projective," in Roshdi Rashed, ed., Mathématiques et Philosophie de l'Antiquité à l'Âge classique (Paris, 1991), 239-285; Kirsti Andersen, "Desargues' method of perspective," Centaurus, 34 (1991), 44-91; James Elkins, The Poetics of Perspective (Ithaca/London, 1994), 3.

${ }^{54}$ Joseph Ben David, Éléments d'une sociologie historique des sciences (Paris, 1997), 45-92.

${ }^{55}$ En 1573, Danti fait paraître sa traduction de l'optique d'Euclide qu'il intitule justement La Prospettiva di Euclide. En attestent aussi les concepts issus de l'optique comme piramida visiva ou raggio centrico. Certains 
problèmes perspectifs ont été directement informés par la géométrie. C'est le cas de la perspective du cercle, débitrice des recherches sur les coniques d'Apollonios, cf. Roshdi Rashed, Geometry and Dioptrics in Classical Islam (London, 2005).

${ }^{56}$ Le rôle des tierces-disciplines fournissant le modèle d'une mathématisation aboutie à une autre discipline est étudié par Roshdi Rashed, "La mathématisation de l'informe dans la science sociale," La Mathématisation des doctrines informes, éd. G. Canguilhem (Paris, 1972), 71-105, cit. 75.

${ }^{57}$ À peine peut-on évoquer l'étrange sténographie arguésienne, Euvres de Desargues, 1, 402, 413.

${ }^{58}$ La quantification explicite n'intervient que sous forme d'exemples numériques de la loi de diminution des grandeurs, par exemple: Piero, De prospectiva pingendi, 73-74.

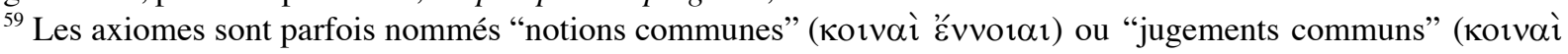
$\left.\delta \sigma^{\prime} \xi \alpha 1\right)$, mettant ainsi l'accent sur le consensus. "Les Axiomes sont à part: en effet, il sont nécessaires par soi [...] nerf de la démonstration, ce sont des médiateurs entre les étapes du raisonnement, à qui ils communiquent leur caractère de nécessité [...] Le Postulat a un statut hybride: si une procédure de décision existait quant à sa démontrabilité, il basculerait aussitôt soit du côté des théorèmes, soit du côté des axiomes indémontrables, sauf qu'il n'est pas, comme eux, indubitable en soi," Maurice Caveing, "Introduction générale," in Euclide, Les Éléments, 119-120, 123.

60 "Erit ergo pictura intercisio pyramidis visivae secundum datum intervallum posito centro," Leon Battista Alberti, De la peinture/De pictura (1435), ed. Jean-Louis Schefer (Paris, 1992), 102; “Adunque taglierai la piramide...," Vinci, Notebooks, 1, 34. 